COMMENT: I agree with the authors that valproate, a drug with known hepatotoxicity and potentially fatal side-effects, should not be used as a first-line therapy in children with epilepsy. Also, its use in the treatment of febrile convulsions seems unacceptable. Despite the less worrisome estimates of fatalities from the US (1 in 10,000; 1 in 7,000 for polytherapy and 1 in 37,000 for monotherapy) 1 and from England ( 1 in 20,000$)^{2}$, careful monitoring of valproate therapy should be mandatory. Fatalities for VPA polytherapy in children < $3 \mathrm{yr}$ was 1/500. ('Dreifuss FE, Santilli N. Neurology 1986;36 (Suppl 1)

:175. Dreifuss FE et al. Neurology 1987;37:379. 2Jeavons PM. Epilepsia 1984; 35 (Supp1 1): $\overline{50-55)}$

CARBAMAZEPINE - EXACERBATED EPILEPSY

Reporting from Denver, Colorado, the authors studied 49 children and adolescents whose seizures reportedly worsened during carbamazepine (CBZ) therapy. In 26 well documented cases, the drug at therapeutic dose levels induced exacerbation of absence, atonic myoclonic and generalized tonic-clonic seizure patterns. The effect was dose-related in 10 patients. Three of 11 patients who had their first absence seizure when CBZ was introduced developed absence status. In addition to childhood absence, the epileptic syndromes wor sened by CBZ included focal symptomatic (frontal lobe), LennoxGastaut, and severe myoclonic epilepsy of childhood. (Horn CS, Ater SB, Hurst DL. Pediatric Neurology 1986;2:340-345)

COMMENT: This is the sixth report concerning seizures induced or exacerbated by carbamazepine. Partial complex seizures are frequently responsive but absence, generalised tonic-clonic, focal, or myoclonic epilepsies may be worsened by CBZ. A slow withdrawal of the CBZ results in improved seizure control.

\title{
BIOTINIDASE DEFICIENCY AND SEIZURES
}

Authors from La Jolla, USA and Florence, Italy report the case of a boy who was first admitted to hospital at 5 years of age because of acute somnolence, alopecia, keratoconjunctivitis and perioral stomatitis associated with lactic acidaemia. At 6 years of age he had grand mal seizures and ataxia and at 7 years he was admitted in coma. The diagnosis of multiple carboxylase deficiency due to biotinidase deficiency was then suspected and treatment with biotin $10 \mathrm{mg}$ p.o.q.d. was started after urine for organic acid analysis was collected. The clinical response was dramatic. Coma and acidosis resolved within a few days. No further seizures occurred. The skin gradually returned to normal and hair, eyebrows and eyelashes regenerated. Ataxia responded after a few weeks but optic atrophy and nerve deafness persisted.

This patient's history began at 6 months of age with seizures. He had multiple generalized tonic-clonic seizures without fever, apparently unresponsive to medication. His subsequent motor development was slow; he sat at 1 year and walked at 2 years. The EEG showed a diffuse abnormality with poorly localized spike foci. Alopecia, a characteristic sign of biotinidase deficiency, did not develop until after 2 years of age. (Thuy LP et al. J. Neurogenet. $1986 ; \underline{3}: 357-363)$

COMMENT: Biotin-related genetically determined disorders are of two types: 1. young infantile or neonatal disease caused by 